\title{
Staged Approach to Thermal Development of a formation with Tar Mat
}

\section{Abdullah Alwazeer, Petroleum Development Oman}

As primary recovery declines in a heavy oil field an EOR process becomes an essential long term field development mechanism. There are several EOR methods to select from and thermal EOR is gaining momentum as experience and successful developments are becoming wide spread. Monitoring and surveillance has also improved in part due to technology advancements to operate in extreme thermal conditions. There is a need to carefully approach a thermal development and this presentation will discuss the staged approach taken to better facilitate the following thermal development. It is believed that a heavy oil field in South Oman has a tar Mat like base (permeability barrier in the oil column) where water in the aquifer is shielded from the producible layer. Analysis work done on viscosity indicated that primary production can be achieved and thermal phase should follow. This was confirmed by trials done on selected wells. Leading to the implementation of a "staged approach" starting with cold recovery, followed by cyclic steam stimulation and then steam drive. The improved plan required a change in surface pipe lines and facility and also well completion designs to accommodate as much as possible both modes of recovery (cold and thermal). This presentation will go over the staged approach for the thermal development and the challenges experienced in getting there. 